\title{
A global monitoring system for electricity consumption and production of household roof-top PV systems in Madeira
}

\author{
Roham Torabi ${ }^{1,2}$ (i) $\cdot$ Sandy Rodrigues ${ }^{2,3} \cdot$ Nuno Cafôfo $^{2} \cdot$ Lucas Pereira $^{2,4} \cdot$ Filipe Quintal $^{2,4}$. \\ Nuno Nunes ${ }^{2,3} \cdot$ Fernando Morgado-Dias ${ }^{2,4}$
}

Received: 5 March 2018 / Accepted: 19 October 2018/Published online: 13 November 2018

(c) Springer-Verlag London Ltd., part of Springer Nature 2018

\begin{abstract}
This paper describes recent work on the development of a wireless-based remote monitoring system for household energy consumption and generation in Madeira Island, Portugal. It contains three different main sections: (1) a monitoring system for consumed and produced energy of residencies equipped with photovoltaic (PV) systems, (2) developing a tool to predict the electricity production, (3) and proposing a solution to detect the PV system malfunctions. With the later tool, the user (owner) or the energy management system can monitor its own PV system and make an efficient schedule use of electricity at the consumption side. In addition, currently, the owners of PV systems are notified about a failure in the system only when they receive the bill, whereas using the proposed method conveniently would notify owners prior to bill issue. The artificial neural network was employed as a tool together with the hardware-based monitoring system which allows a daily analysis of the performance of the system. The comparison of the predicted value of the produced electricity with the actual production for each day shows the validity of the method.
\end{abstract}

Keywords Roof-top PV system · Prediction · Artificial neural network · Monitoring system

\section{Introduction}

Over the last few years, the decrement in the manufacturing cost associated with photovoltaic (PV) systems has affected the electricity industry within various aspects. For example, from utilities points of view, they have become a costcompetitive alternative to conventional power plants, whereas from users points of view, they are an attractive option to reduce the electric bill [1]. The power produced by a PV system depends on the variability of solar irradiance,

Roham Torabi

roham.torabi@m-iti.org

Fernando Morgado-Dias

morgado@uma.pt

1 Faculty of Sciences and Technology, University of Coimbra, Coimbra, Portugal

2 Madeira Interactive Technology Institute, Funchal, Portugal

3 Instituto Superior Técnico, University of Lisbon, Lisbon, Portugal

4 University of Madeira, Funchal, Portugal changing seasons and environmental factors. Intermittent behavior of the output (energy production) of a PV system may increase the operating costs for the electricity system operator by increasing requirements of primary reserves and reducing the reliability of electricity supply. In a scenario in which a considerable portion of the energy of an electric grid is provided by the PV systems, the prediction of the changes on daily production might be necessary in order to schedule the spinning resources capacity [2].

Consequently, the prediction of the generated power and monitoring the consumption are important for planning the operations of the power grid efficiently. In the present age of energy management systems (EMS), intelligent management assistance is no longer an option, but a necessity [3].

Furthermore, small-sized grid-connected PV systems are often not monitored, because of the high monitoring system costs. In practice, the owners of small-scale PV system (households) may notice there is a problem in the PV system when the production is completely down or the energy production value as shown in the bill is wildly different than the expected value. Nevertheless, there are more reasons as motivation to make a performance 
analyzer for monitoring the PV systems, for instance, increasing the reliability of the electricity supply and improving the quality of scheduling the network operations, particularly in isolated grids where reserves are limited. The PV system performance analyzer (PA) enables the produced energy to be used more efficiently, depending on the employed objective functions.

This work has been performed under the scope of SmartSolar Project, in Funchal the capital of Madeira island, Portugal. The actual and forecast daily solar radiation values were provided by IPMA (Instituto Português do Mar e da Atmosfera). The corresponding data used in this work are gathered from the mentioned companies.

Regarding the main policies and legislation associated with PV system installation, the Portuguese law indicates that the PV system installations in the residential sector must include two electricity meters, namely the household consumption meter and the PV solar production meter [4]. Hence, a hardware (the SmartSolar-Box) was built that measures and stores both the consumption and production values to then analyze the data to detect some abnormalities. The proposed solution combines a monitoring system with an artificial neural network (ANN) model, interacting with an algorithm that analyzes the values provided by the ANN model. The algorithm can be used to detect PV system malfunctioning through a simple comparison of the daily production value with the estimated one. If the predicted values are outside the defined threshold during five consecutive days, thus, the system emits an alarm message.

This paper is organized as follows: In Sect. 2, the methodology is described, followed by the description of PV system performance analyzer (PA). The SmartSolar project is explained in Sect. 3. The statistical method used for developing an estimator as tool is described in Sect. 4. This section was published as a conference proceeding in ES2DE 2017, Madeira, Portugal [5]. The assumptions and several scenarios are presented in Sect. 5 where the results are shown. The main conclusion and future work are discussed in Sect. 6.

\section{Methodology}

Figure 1 illustrates various parts of the performance analyzer (PA) in which the interaction between the components is drawn. The PA is divided into four parts:

I. The production data,

II. The solar radiation data,

III. The artificial neural network model and

IV. The performance analyzer.

The SmartSolar-Box receives the solar production values and sends it to a database through an Ethernet or Wi-Fi connection (details are given in Sect. 3). The required input for the ANN model is the solar radiation value in $\mathrm{kJ} / \mathrm{m} 2$. This solar radiation value is given by IPMA. Thereafter, PA determines whether the system is functioning properly or not. It receives two daily production inputs in $\mathrm{kWh}$ from the same installation: one of them is the daily production which comes from the SmartSolar-Box and the other comes from the ANN model. To do so, since the predicted value contains an error (it might be estimated higher or less), therefore, a threshold on top of the estimated value is introduced.

\subsection{Threshold and alarm}

To make an alarm system, the differences between the outputs and the targets are analyzed for setting the alarm threshold. An average of the value of the targets is calculated; thus, $15 \%$ of its absolute value is added to and subtracted from the estimated value (output) to make the upper and lower boundaries, where the outputs are taking place (15\% was chosen on an empirical basis after a trial and error procedure). If both the estimated value and the target are inside the predefined interval, it means that there is no failure in the PV system.

This is performed to account for the uncertainty in the model regarding different locations of meteorological stations and PV systems. On the other hand, if there is a considerable difference between the estimated value and target such that it crosses the thresholds boundaries, it means the system is not functioning properly. In addition, if this happens for five days in a row, an alarm would be issued to inform the responsible sector of a malfunctioning in the PV system. It is necessary to use a set of days in a row (in this work five days are considered) to assure the problem has not arisen from inaccuracies in the estimator or due to sudden changes in weather circumstances. More details with examples are given in Sect. 5.1.

\section{SmartSolar-Box}

The SmartSolar monitoring system consists of the SmartSolar-Box, which is connected to the two energy meters as shown in Fig. 2. The SmartSolar-Box measures the energy of both meters through a current and voltage sensor. It can calibrate itself using an impulse sensor if a smart meter is available in place for the consumption side. To collect data for monitoring the consumption and production, the appropriate hardware was chosen to preprocess, store and send data cost-effectively to a remote database. The SmartSolar-Box is built based on a BeagleBone board.

Most of the proposed Monitoring systems [6-8] used the Arduino board. However, in the SmartSolar-Box, 


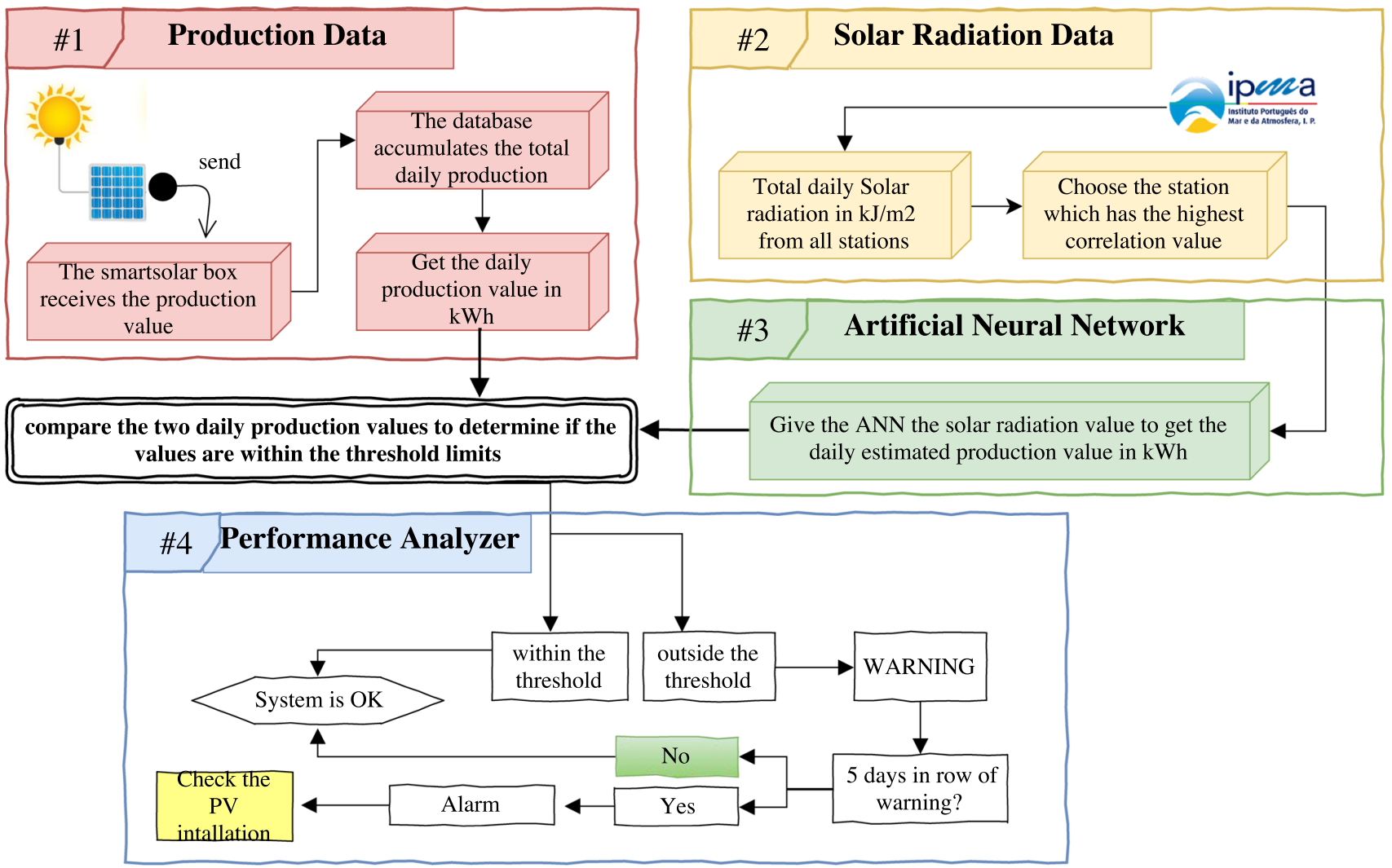

Fig. 1 Flowchart of the PA procedure

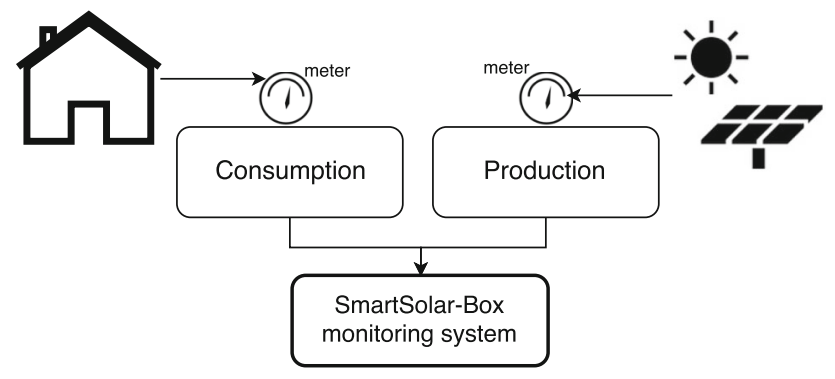

Fig. 2 SmartSolar-Box concept

BeagleBone board was used because of its advanced features. These features include more analog inputs (impulse sensors and current sensors), CPU with Faster speed (allows small sampling times ex: 4000 samples per second) and two Programmable Real-time Unit (PRU). The SmartSolar-Box consists of a BeagleBone Black embedded single board computer, with six $3.5-\mathrm{mm}$ jacks, three RJ11 connectors, one RJ45 connector, one USB connector that is connected to a $\mathrm{Wi}-\mathrm{Fi}$ dongle and a $230 \mathrm{~V}$ power connector to feed the board. Table 1, Figs. 3 and 4 represent the hardware and its connections.

Two of the 3.5-mm jacks are used to connect the consumption and production current sensors, and the voltage sensor is connected directly to the board, while the rest of
Table 1 Front and rear inputs specification

\begin{tabular}{llll}
\hline & Key & Type & Pin \\
\hline Front input & Production impulse sensor & Digital & P9_16 \\
& Consumption impulse sensor & Digital & P9_13 \\
& Production current sensor & Analog & AIN5 \\
& Inactive & Analog & AIN5 \\
& Unused 0 & Digital & P9_13 \\
Rear input & Consumption current sensor & Analog & AIN6 \\
& Radiation sensor & Analog & AIN1 \\
& Unused 1 & Analog & AIN0 \\
& Unused 2 & Analog & AIN2 \\
\hline
\end{tabular}

the $3.5-\mathrm{mm}$ connectors are free to add other sensors, for instance, a solar radiation sensor. The BeagleBone receives the sensor information every second to calculate the consumption and production power every minute. These power calculations are sent to the database through the Ethernet cable or Wi-Fi dongle or stored in the internal memory in case of connection failure. The power is calculated with every instant current and voltage point (not rms) which translates into 4000 samples per second, which corresponds to 80 points per wavelength in a $50-\mathrm{Hz}$ grid. 
Fig. 3 SmartSolar-

Box hardware
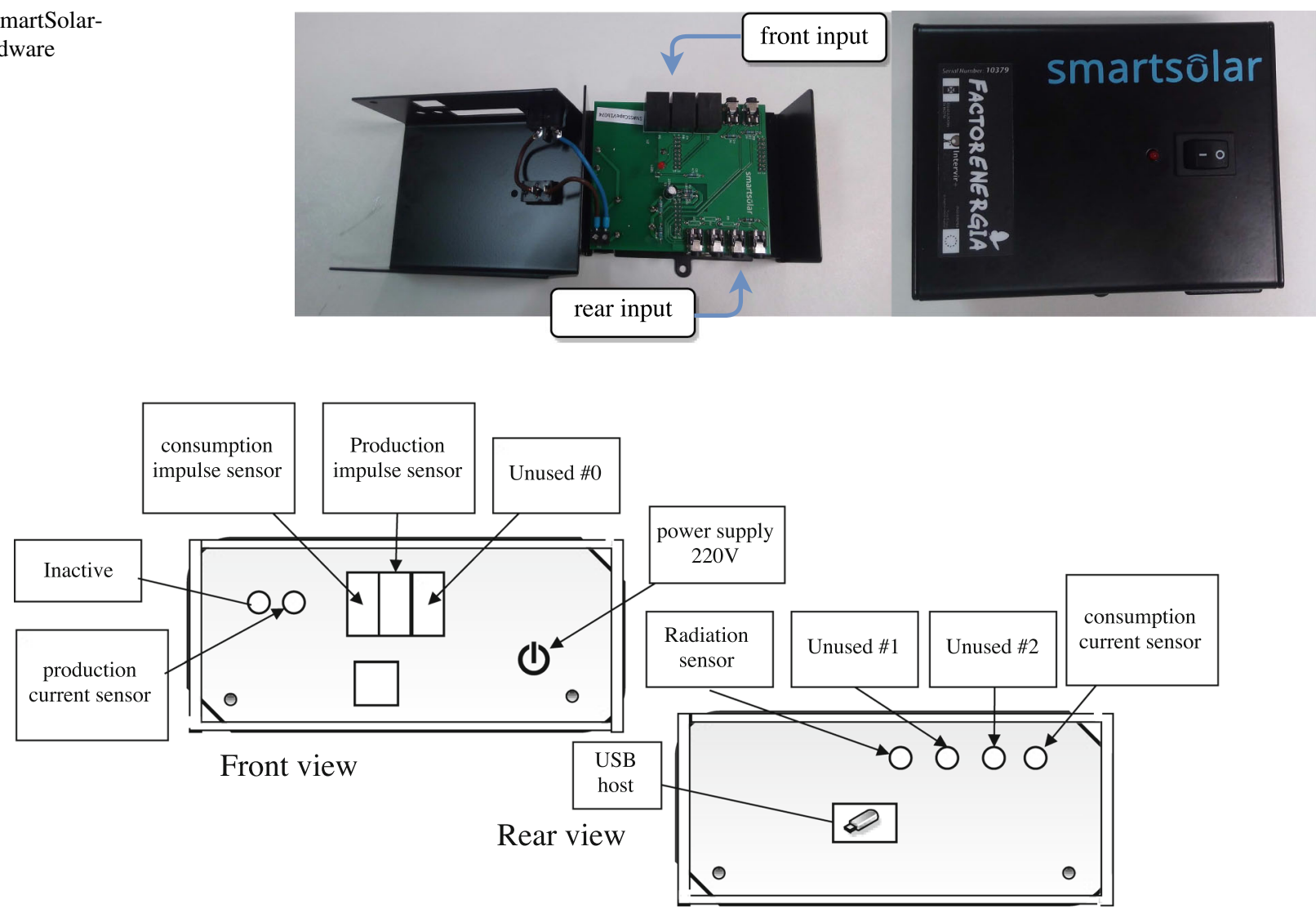

Fig. 4 Front and rear views of the hardware

\section{Estimator}

The estimation of the PV production is associated with the weather conditions and forecasts. The estimated output is converted into electric energy $(\mathrm{kWh})$. The existing solutions in the literature might be categorized into the three types of physical, statistical and hybrid methods [9].

I. Physical models are simple, based on the global irradiance on the solar cell, or they may be more complex if additional parameters are included. The electricity produced by PV modules is affected by several factors such as solar irradiance and shadowing, soiling, aging, cell temperature, the solar incidence angle and the load condition. Hence, it is not easy to predict the produced energy due to the conversion process dependency to several parameters as ambient and cells conditions.

II. Statistical methods are mainly based on the concept of stochastic time series or persistence. Many researchers have been working on providing a forecasting tool in order to predict PV power production, where the use of machine learning methods has become the most common approach to forecast a time series future values [10].
III. Hybrid models are combined by two or more of the previously described methods. To improve the forecast value, different models with unique features may be employed. For example, Ogliari et al. proposed a novel hybrid evolutionary approach for training artificial neural networks to achieve more accurate forecasting of PV systems based on weather forecast as input data [11].

Many researchers have been working on providing a forecasting tool to predict PV power production, where most of them have used the two-phase approach. In the first phase, the solar irradiance on different time scales is forecast based on artificial neural networks, fuzzy logic and/or hybrid system [2]. In [12], the authors used recurrent ANN to predict irradiance by using only historical data. In the second phase, the forecast irradiance data are used as inputs in commercial PV simulation software, such as TRNSYS [13] and HOMER [14]. The output of these programs is an estimated simulation, on an hourly basis of the AC power production of the PV system.

Chen et al. [2] introduced an ANN that uses a radial basis function, as an activation function, in order to forecast $24 \mathrm{~h}$ ahead of power generation of a roof-top PV system for an experiment in online training. Rather than 
using two-phase methods as described previously, authors made a direct forecast of the output power of the PV system from its historical record and the online meteorological services.

The reviewed literature confirms that artificial neural networks have been successfully applied for forecasts of intermittent energy supply, particularly of PV production [2, 15-19]. The ANNs, as illustrated in Fig. 5, are composed of simple elements operating in parallel which are inspired by the biological nerves systems. ANNs can be trained to perform a function by adjusting the values of connections (weights) between the elements. Typically, ANNs are trained so that an input leads to a specific target output. In the figure below, the network is adjusted, based on a comparison of the input and the target. Typically, a considerable number of input/target pairs are required to train a network. In this paper, the daily irradiance and the daily electricity production are considered as input and target pairs.

In order to make the prediction tool, a multilayer perceptron (MLP) was employed that maps sets of inputs (irradiance) onto a set of appropriate outputs (daily production). An MLP consists of multiple layers of nodes in a directed graph and each layer fully connected to the next one. Figure 6 represents the structure of the ANN used in this work. Except the input, each node is a neuron (or processing element) with a nonlinear activation function. The MLP utilizes a supervised learning technique called back-propagation to train the network. As it is shown, it consists of an input, two layers of neurons (one hidden and one output layer) and an output. The hidden layer contains four neurons with the transfer function, tan-sigmoid [20]. Equation (1) demonstrates the mathematical explanation of tan-sigmoid.

$a=\operatorname{tansig}(n)=\frac{2}{1+e^{(-2 \times n)}}-1$

where $\boldsymbol{n}$ is the input and $\boldsymbol{a}$ represents the output. It calculates the output layer according to its net input. The data were manually introduced into the $\mathrm{ANN}$, and then, the training phase was applied. For every month, the data were sorted randomly, and then, out of each month (30 days)

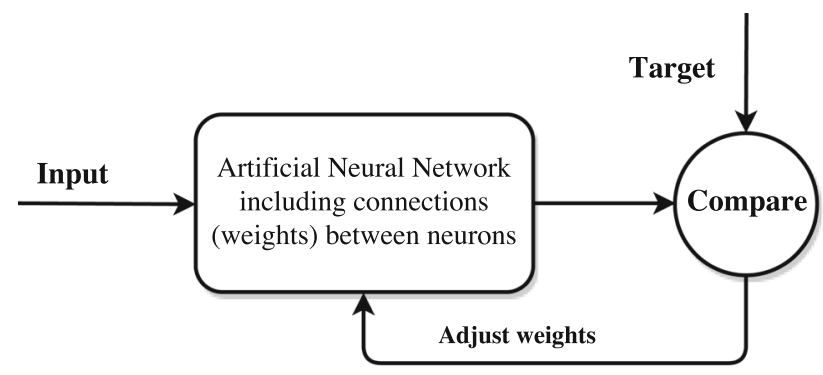

Fig. 5 Artificial neural networks overview [20]
$\% 70$ of the samples were selected for training, $\% 15$ for validation and $\% 15$ for testing phases. In the training phase, the Levenberg-Marquardt back-propagation method was employed, which is a function that updates weight and bias values according to Levenberg-Marquardt optimization method [21].

In the following, the data used in this work is presented, followed by the description of the scenarios and considered assumptions. The daily accumulated irradiance magnitude $\left(\mathrm{kJ} / \mathrm{m}^{2}\right)$ is available from several meteorological stations, for the period of 2014 to 2015 . In addition, the historical data of the accumulated daily production (energy in $\mathrm{kWh}$ ) for each of the PV systems had been recorded within the same time frame (from 2014 to 2015). For the samples used for this work, in most of the cases, the size of the PV systems do not exceed more than $5 \mathrm{~kW}$ installed power, except in one case in which the installed power is $12 \mathrm{~kW}$. Figure 7 shows both the geographical location of all the considered PV systems and the location of the meteorological stations (three of them). Madeira island is in Atlantic Ocean where its capital Funchal has coordinates of $32^{\circ} 39^{\prime} \mathrm{N} 16^{\circ} 55^{\prime} \mathrm{W}$ based on numerical coordination system.

Most of the PV systems are located in the south and southwest of the island. There are fourteen meteorological stations that spread out across the island. In this work, the data of only the three of them were used (as the input) to have the best station and the other two as backup in case of lack of the data from the prior one. The selection process of these three among the other meteorological stations is as follows:

I. Firstly, the correlation between the data of produced energy (daily $\mathrm{kWh}$ ) and the value of the irradiance $\left(\mathrm{kJ} / \mathrm{m}^{2}\right)$ from all the meteorological stations was calculated. Then, the meteorological stations with the highest correlation values were selected. In addition, the values from the meteorological stations, those which are ranked as the second and third as the most correlated stations, are reserved as backup values in case of not accessing to the data from assigned (the first) weather station.

II. This procedure was applied to all the PV installations individually. The consequence was a selection of three meteorological stations which are marked as white pins in Fig. 7, namely I. Lugar de Baixo, II. Funchal Observatory and, III. Funchal Lido.

Figure 8 represents the data dispersion over a one-year term (i.e., the daily energy production in $\mathrm{kWh}$ ). It is worth mentioning that a significant portion of the data was missing, which caused series of obstacles in the result. Within a one-year term (365 days) for the 24 PV installation points, 8760 samples would be an ideal number to develop a prediction tool for the case study of this research. 
Fig. 6 Common neural network structure [20]

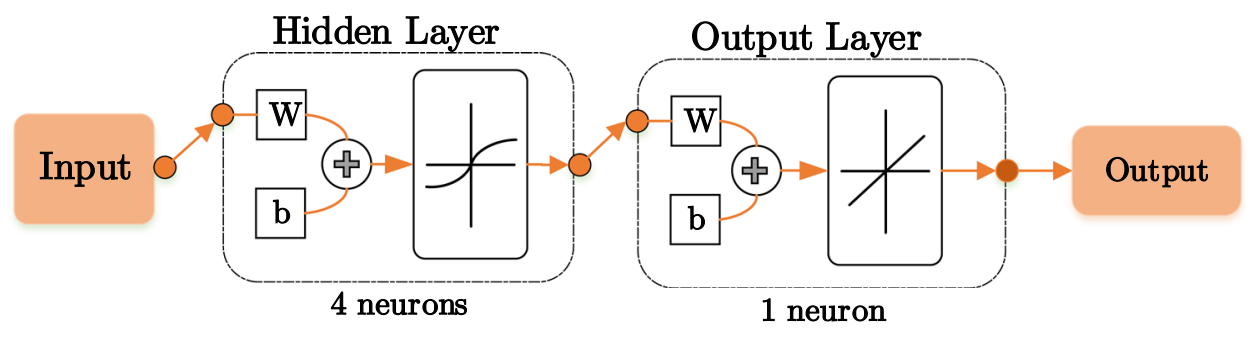

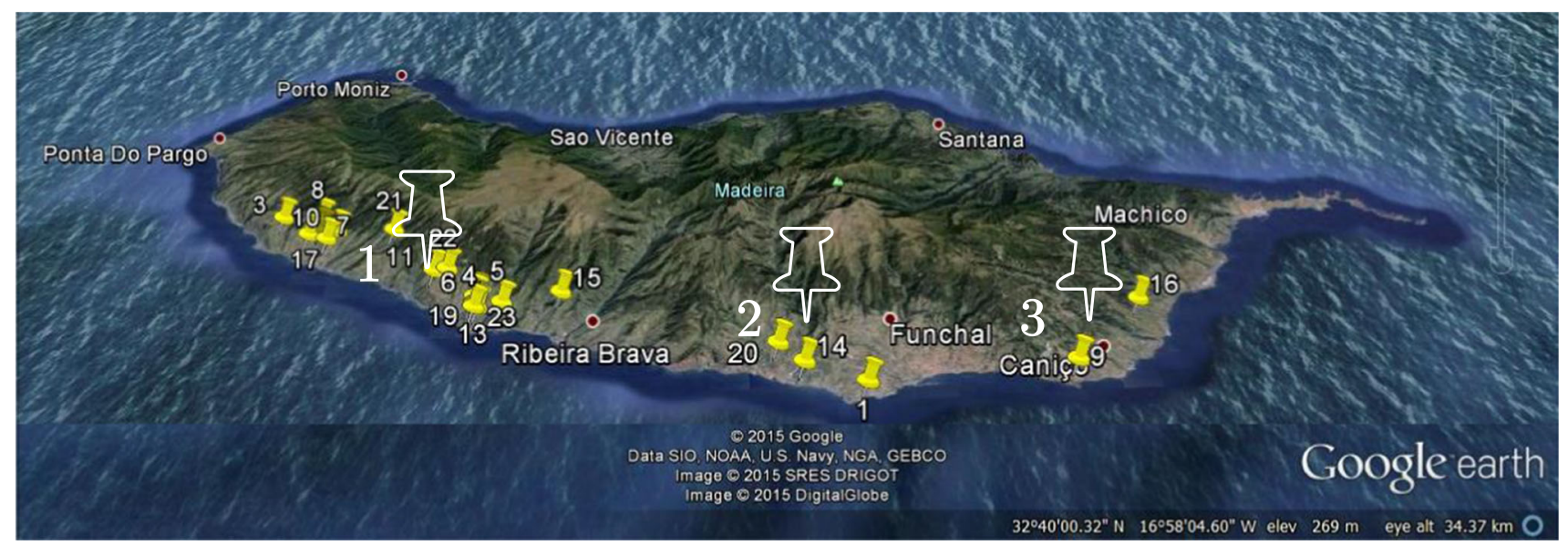

Fig. 7 Geographical location of PV systems on Google Maps (yellow pins) and meteorological stations (white pins)

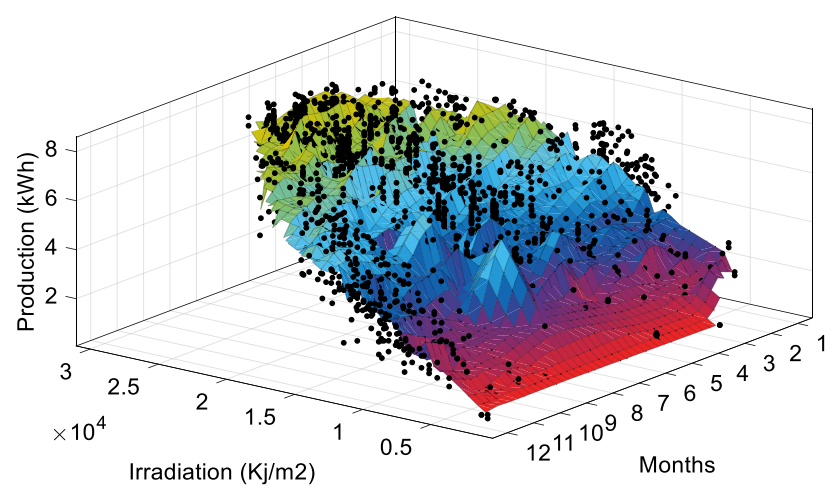

Fig. 8 Data dispersion

However, there were only 3012 samples recorded for all the PV installations (8760 samples are considered as a sufficient number in a scenario in which each PV installation contains 365 days, of record). Nevertheless, as the result shows, the predicted values from the model are slightly differed from the actual values.

\subsection{Models}

The three main models are defined as follows:

I. One Generic model.

II. Three Localized models for the three meteorological stations mentioned earlier

\section{Four Specific models}

The Specific model corresponds to a model which was built by pairs of inputs and targets from only one PV installation system which had the highest correlation with one of the meteorological stations. As shown in Fig. 7, three different locations are highlighted. In all of them, an ANN as a tool is employed in order to generate the value of the produced energy (kWh) based on the solar radiation values. The result is shown in the next section.

\section{Evaluation and result}

The purpose of making both models (Localized and Specific), was to examine the accuracy of the Generic model. Hence, the performance of each model was assessed. The result of this evaluation is presented in Figs. 9 and 10 , for the Specific and Localized models, respectively. The result for the Generic model is shown in Fig. 11. The outcome of this evaluation is drawn in four separated graphs as follows:

- The function-fit (the up-left graph) shows the errors, through drawing the differences between the targets (the real production values) and outputs (estimated values).

- The correlation (R) between the targets and outputs is demonstrated in the right upper part of each figure. 
Fig. 9 Result of the performance of the Specific Model. It is called Conduminho

Fig. 10 Result of the performance of the Localized model (Lugar de baixo (1))
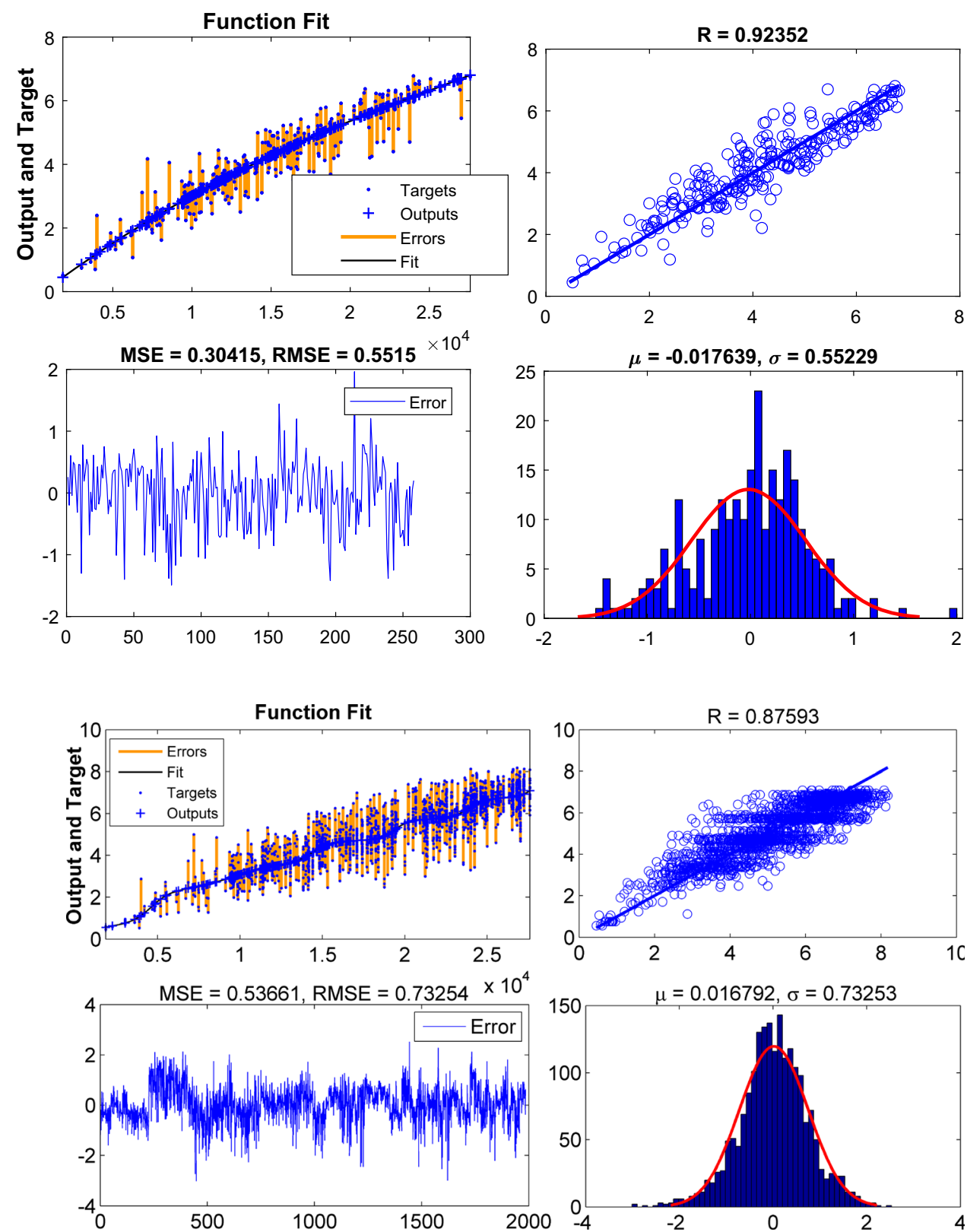

- The mean squared error (MSE) measures the average of squares of the error that is the difference between the estimated output and the real production (target).

- And finally, the right down part of the figure shows the histogram of errors, along with the mean and standard deviation.

Figure 9 represents the result of the Specific model, made by using 250 samples from only one PV system which is called Conduminho. Figure 10 shows the result of an evaluation of employing the Localized model, made by using 2056 samples gathered from $17 \mathrm{PV}$ installations. Figure 11 demonstrates the result of the same assessment for the Generic model, using all the data (3012 samples).
A comparison between the Generic model, three Localized and four Specific models was made. The same pairs of inputs and targets (samples that were not used in the training phase of any ANN models) of four PV system installations (namely Club Naval, Antonio, Conduminho and Neto) were given to all the models. Afterward, the values for each of the correlations $(R)$, mean square error $(M S E)$ and root-mean-square error (RMSE) were calculated. Table 2 presents these values all together.

As shown in Table 2, the R, MSE and RMSE associated with each model in comparison with the answers (outputs) given by the Generic model are slightly different. Therefore, it can be concluded that the answers given by the Generic model are acceptable. 
Fig. 11 Performance of the Generic model
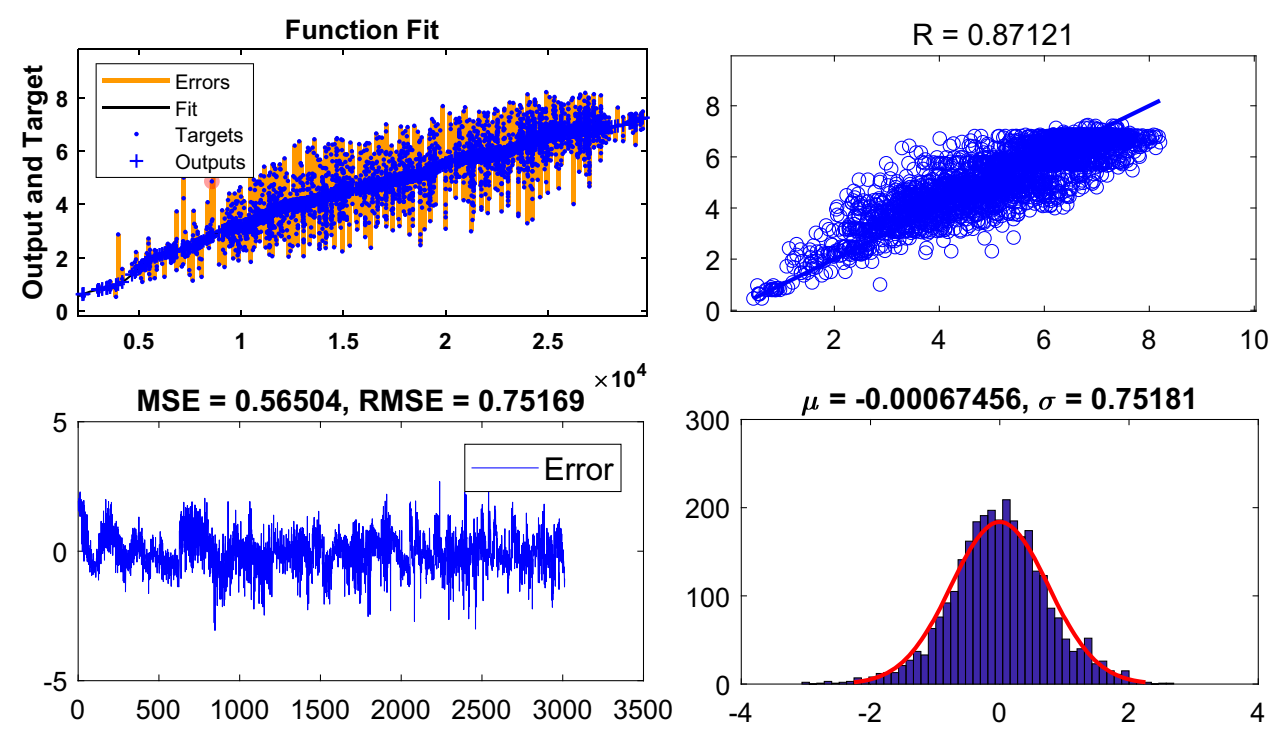

Table 2 Comparison between Generic, Localized and Specific Models

\begin{tabular}{|c|c|c|c|c|c|c|c|c|c|}
\hline \multirow{2}{*}{$\begin{array}{l}\text { Model } \\
\text { Locations }\end{array}$} & \multicolumn{3}{|c|}{ Specific } & \multicolumn{3}{|c|}{ Localized } & \multicolumn{3}{|c|}{ Generic } \\
\hline & $\mathrm{R}$ & MSE & RMSE & $\mathrm{R}$ & MSE & RMSE & $\mathrm{R}$ & MSE & RMSE \\
\hline 3 (Club Naval) & 0.88 & 6.40 & 2.50 & 0.87 & 7.10 & 2.70 & 0.84 & 10.4 & 3.20 \\
\hline 3 (Antonio) & 0.94 & 3.20 & 1.80 & 0.93 & 3.40 & 1.90 & 0.93 & 4.00 & 2.00 \\
\hline 1 (Condominio) & 0.92 & 53.60 & 7.30 & 0.92 & 69.70 & 8.40 & 0.92 & 59.00 & 7.70 \\
\hline 2 (Neto) & 0.89 & 5.70 & 2.40 & 0.89 & 5.80 & 2.40 & 0.90 & 5.40 & 2.30 \\
\hline
\end{tabular}

Furthermore, the following figures illustrate the trend of each model in computed outputs through feeding them with bunch of homogeneous samples (inputs). The answers given by each model were compared to the targets, as Figs. 12 and 13 demonstrate this comparison. Each figure shows a series of answers (30 days) given by the Specific, Localized and Generic models.

In each graph, the target represents the actual value of the production, which was recorded previously. The answers given by Generic, Localized and Specific models represent the outputs using different ANNs as the estimator. Figure 13 shows this comparison for the installation named Club Naval (4.1 kW of installed power) located in zone three.
In most of the cases, all the three models (Generic, Localized and the Specific) give the outputs which are very close to the targets. It is worth mentioning that due to having sufficient pair of samples (about 2000 samples) for the zone one, more samples were participated in the training phase of building up the models. As a result, the Generic model shows a better performance for the installations which are in this zone.

\subsection{Threshold and alarm}

As shown in Table 2, in most of the cases, the outputs given by the Specific model are closer to the targets followed by the answers from the Local Generic model and at

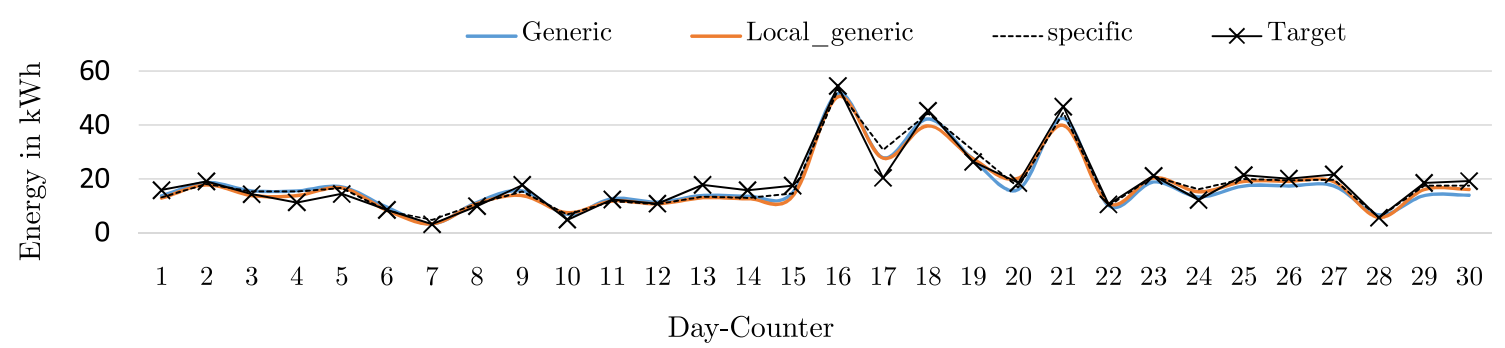

Fig. 12 Target Vs the outputs given by Generic, Localized and Specific models from zone 1 (Neto) for the month of February 


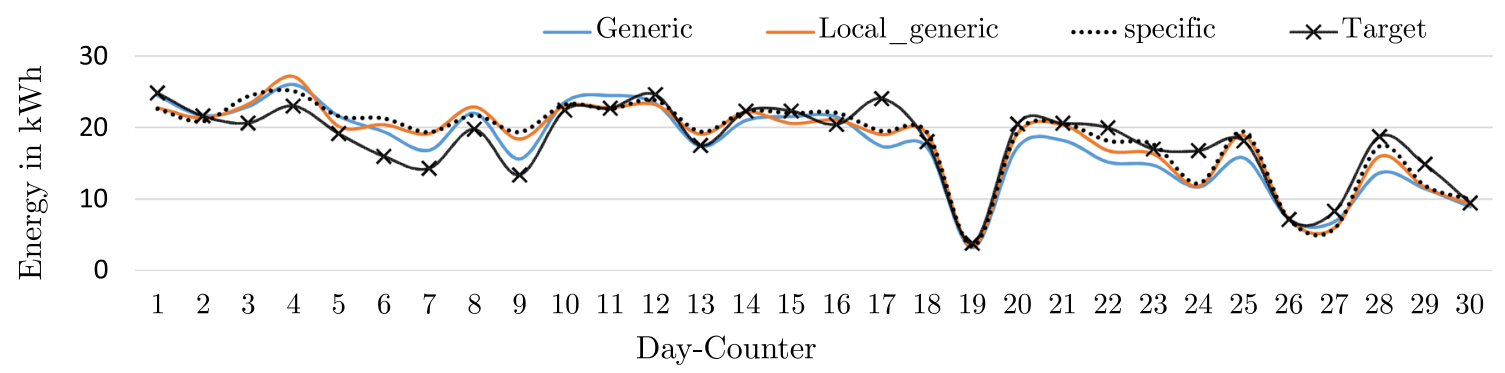

Fig. 13 Target Vs the outputs given by Generic, Localized and Specific models from zone 3 (Club Naval), for the month of September

last the Generic model, respectively. In order to facilitate the prediction procedure and be able to use the Generic model as the main estimator for all the PV systems, in this work, a threshold was defined. Figure 14 demonstrates the answers estimated by the Generic model, the targets and the defined domain of the alarm threshold for a period of thirty days. If both of the estimated value and the target are inside the domain (between the Generic-min and Generic$\max$ ), it means that there is no failure in the PV system. On the other hand, if there is a considerable difference between the estimated value and target such that the target is out of the threshold' $\mathrm{s}$ boundaries, it means there is a malfunctioning in the PV system. If this happens for five days in a row, consequently an alarm would be issued to inform the authorities about the failure. It is necessary to use a set of days in a row (in this work five days was considered) to assure that the problem is not arisen from inaccuracies in the estimator or due to sudden changes in the weather circumstances.

\section{Conclusion}

This work includes different parts of the SmartSolar project: i) the SmartSolar-Box as a hardware was introduced. It is responsible to collect, store and send the data (the values of energy consumption and energy production) to a receiver center, ii) the artificial neural network was employed in order to develop a tool to predict the produced energy by roof-top PV systems and iii) the last part focused on developing a model as the PV performance analyzer, using the outputs given by the ANN model, in order to detect the malfunctioning arisen from PV systems.

One of the significant obstacles in this work lies in the very particular orography of Madeira island. In addition, its sudden weather changes affects the accuracy of the estimated value of the energy production. This is more of a concern for those PV systems which are not located close by their associated weather stations. Consequently, the authors tried to make various models (Generic, Localized and Specific models) and then by comparing the given outputs from each model with the targets studied the factors which are involved in the values of the outputs. For instance, it was perceived that for the two PV installations which are located in a same zone, the altitude differences in these two points with their associated weather station has a direct correlation with the accuracy of the estimated value of the produced energy. In other words, the more difference in the altitude, the bigger error in the output.

However, there were some cases that even though they were located in different altitude in comparison with their associated weather stations, the output given by the models contained a very little errors. In conclusion, it is worth mentioning that, beside the influence that the altitude differences may have in the outputs, the quantity of the samples (the more the better) which were participated in the learning phase of building the ANN models, the angle of the solar panel orientation and the cleanliness of the

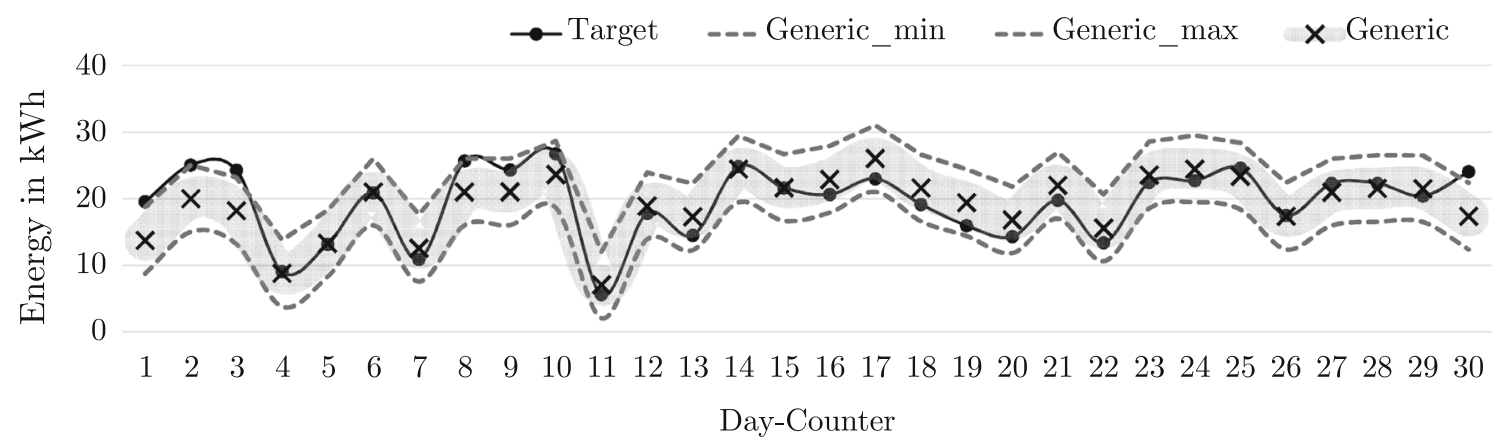

Fig. 14 Estimated values vs targets in the defined amplitude of the alarm threshold 
panels would have a significant contribution in the accuracy of the estimator.

The final goal in this work was to find the most appropriate model by comparing the outputs from the three different models. It was found out that the most accurate answers were given by the Specific model, followed by the Generic Local and Generic model, respectively. However, the histogram of the errors associated with each model showed how far the estimated answers were from the targets, where the answers from the Generic model were not that much far from the outputs given by the other models.

In fact, having the most accurate answer was not the main goal of developing such PA, but rather one common generalized model which can estimate the values of produced energy for all the PV systems. The answers given by the Generic model meet the requirement of the PA, as one of its inputs. The use of the Generic model simplifies the use of the estimator model as well. In other words, it is not necessary to use the Specific models which are assigned to every PV installation system.

Heretofore, the users have not been notified that their roof-top PV system has been or has not been working properly, unless they receive the periodically bill of their production. The hardware-based monitoring system, the developed ANN models, together with the PA, enable a daily monitoring of the PV system performance.

Acknowledgements This work was supported partially by the Funding Program + Conhecimento II, Incentive System to Research and Technological Development and Innovation of Madeira Region II, SmartSolar project MADFDR-01-0190-FEDER-000015.

This work was also supported by Agência Regional para o Desenvolvimento e Tecnologia through the PhD Studentship, Project M1420-09-5369-000001 and by the Portuguese Foundation for Science and Technology through Projeto Estrategico LA9 - UID/ EEA/ $50009 / 2013$

\section{References}

1. Rodrigues S, Torabikalaki R, Faria F, Cafofo N, Chen X, Ivaki AR, Mata-Lima H, Morgado-Dias F (2016) Economic feasibility analysis of small scale PV systems in different countries. Solar Energy 131:81-95

2. Chen C, Duan S, Cai T, Liu B (2011) Online 24-h solar power forecasting based on weather type classification using artificial neural network. Solar Energy 85(11):2856-2870

3. Schmalensee R (2016) The Future of the US Electric Grid. In: Perspectives on Complex Global Challenges: Education, Energy, Healthcare, Security and Resilience, pp. 73-79, Hoboken, New Jersey: John Wiley \& Sons, Inc
4. Ministério do Ambiente Ordenamento do Território e Energia, Decreto-Lei no 153/2014 de 20 de outubro, Diário da República, pp. 5298-5311, 2014

5. Torabi R, Rodrigues S, Cafofo N, Morgado-Dias F (2017) Development of an ANN model to predict the electricity produced by small scale roof-top PV systems in Madeira Island, in Energy and Sustainability in Small Developing Economies, ES2DE 2017 - Proceedings

6. Thomas MS, Bansal P, Taneja P (2014) Smart home energy management by Demand Response controller design, in Power Electronics (IICPE). In: 2014 IEEE 6th India international conference on, pp 1-6

7. Nkoloma M, Zennaro M, Bagula A (2011) SM2: solar monitoring system in Malawi. ITU Kaleidoscope 2011:4-8

8. Luca G, Benedetta M, Nardecchia F, Bisegna F, Chiara G (2015) Home smart grid device for energy saves and failure monitoring. In: 2015 IEEE 15th international conference on environment and electrical engineering, EEEIC 2015 - conference proceedings

9. Ulbricht R, Fischer U, Lehner W, Donker H (2013) First steps towards a systematical optimized strategy for solar energy supply forecasting. In: ECML/PKDD 2013, 1st international workshop on data analytics for renewable energy integration (DARE)

10. Dolara A, Leva S, Manzolini G (2015) Comparison of different physical models for PV power output prediction. Solar Energy 119:83-99

11. Ogliari E, Grimaccia F, Leva S, Mussetta M (2013) Hybrid predictive models for accurate forecasting in PV systems. Energies 6(4):1918-1929

12. Yona A, Senjyu T, Funabashi T (2007) Application of recurrent neural network to short-term-ahead generating power forecasting for photovoltaic system. In: 2007 IEEE power engineering society general meeting, PES

13. Alamsyah TMI, Sopian K, Shahrir a (2004) Predicting average energy conversion of photovoltaic system in Malaysia using a simplified method. Renew Energy 29:403-411

14. Dalton GJ, Lockington D a, Baldock TE (2009) Feasibility analysis of renewable energy supply options for a grid-connected large hotel. Renew Energy 34(4):955-964

15. Bacher P, Madsen H, Nielsen HA (2009) Online short-term solar power forecasting. Solar Energy 83(10):1772-1783

16. Fernandez-Jimenez LA, Mu noz-Jimenez A, Falces A, MendozaVillena M, Garcia-Garrido E, Lara-Santillan PM, Zorzano-Alba E, Zorzano-Santamaria PJ (2012) Short-term power forecasting system for photovoltaic plants. Renew Energy 44:311-317

17. Mellit A, Pavan AM (2010) Performance prediction of $20 \mathrm{kWp}$ grid-connected photovoltaic plant at Trieste (Italy) using artificial neural network. Energy Convers Manag 51(12):2431-2441

18. zgi E, Öztopal A, Yerli B, Kaymak MK, ahin AD (2012) Shortmid-term solar power prediction by using artificial neural networks. Solar Energy 86(2):725-733

19. Hocaolu FO, Gerek ÖN, Kurban M (2008) Hourly solar radiation forecasting using optimal coefficient 2-D linear filters and feedforward neural networks. Solar Energy 82(8):714-726

20. The MathWorks Inc, Neural Network Toolbox, 2017

21. Yu H, Wilamowski BM (2011) Levenberg-Marquardt training, Industrial Electronics Handbook, vol 5. Intelligent Systems, Kyoto, pp 12-18 